\title{
Learning Multi-dimensional Indexes
}

\author{
Vikram Nathan*, Jialin Ding*, Mohammad Alizadeh, Tim Kraska \\ \{vikramn,jialind,alizadeh,kraska\}@mit.edu \\ MIT CSAIL
}

\begin{abstract}
Scanning and filtering over multi-dimensional tables are key operations in modern analytical database engines. To optimize the performance of these operations, databases often create clustered indexes over a single dimension or multidimensional indexes such as R-Trees, or use complex sort orders (e.g., Z-ordering). However, these schemes are often hard to tune and their performance is inconsistent across different datasets and queries. In this paper, we introduce Flood, a multi-dimensional in-memory read-optimized index that automatically adapts itself to a particular dataset and workload by jointly optimizing the index structure and data storage layout. Flood achieves up to three orders of magnitude faster performance for range scans with predicates than state-of-theart multi-dimensional indexes or sort orders on real-world datasets and workloads. Our work serves as a building block towards an end-to-end learned database system.
\end{abstract}

\section{CCS CONCEPTS}

- Theory of computation $\rightarrow$ Data structures and algorithms for data management; • Information systems $\rightarrow$ Database query processing; Data analytics.

\section{ACM Reference Format:}

Vikram Nathan*, Jialin Ding*, Mohammad Alizadeh, Tim Kraska. 2020. Learning Multi-dimensional Indexes. In 2020 ACM SIGMOD International Conference on Management of Data (SIGMOD'20), Fune 14-19, 2020, Portland, OR, USA. ACM, New York, NY, USA, 16 pages. https://doi.org/10.1145/3318464.3380579

\section{INTRODUCTION}

Scanning and filtering are the foundation of any analytical database engine, and several advances over the past several

\footnotetext{
${ }^{\star}$ Equal contribution.

Permission to make digital or hard copies of all or part of this work for personal or classroom use is granted without fee provided that copies are not made or distributed for profit or commercial advantage and that copies bear this notice and the full citation on the first page. Copyrights for components of this work owned by others than the author(s) must be honored. Abstracting with credit is permitted. To copy otherwise, or republish, to post on servers or to redistribute to lists, requires prior specific permission and/or a fee. Request permissions from permissions@acm.org. SIGMOD '20, June 14-19, 2020, Portland, OR, USA

(๑) 2020 Copyright held by the owner/author(s). Publication rights licensed to ACM.

ACM ISBN 978-1-4503-6735-6/20/06 .. \$15.00

https://doi.org/10.1145/3318464.3380579
}

years specifically target database scan and filter performance. Most importantly, column stores [7] have been proposed to delay or entirely avoid accessing columns (i.e., attributes) which are not relevant to a query. Similarly, there exist many techniques to skip over records that do not match a query filter. For example, transactional database systems create a clustered B-Tree index on a single attribute, while column stores often sort the data by a single attribute. The idea behind both is the same: if the data is organized according to an attribute that is present in the query filter, the execution engine can either traverse the B-Tree or use binary search, respectively, to quickly narrow its search to the relevant range in that attribute. We refer to both approaches as clustered column indexes.

If data has to be filtered by more than one attribute, secondary indexes can be used. Unfortunately, their large storage overhead and the latency incurred by chasing pointers make them viable only for a rather narrow use case, namely when the predicate on the indexed attribute has a very high selectivity; in most other cases, scanning the entire table can be faster and more space efficient [6]. An alternative approach is to use multi-dimensional indexes; these may be tree-based data structures (e.g., k-d trees, R-Trees, or octrees) or a specialized sort order over multiple attributes (e.g., a space-filling curve like Z-ordering or hand-picked hierarchical sort). Many stateof-the-art analytical database systems use multi-dimensional indexes or sort-orders to improve the scan performance of queries with predicates over several columns. For example, both Redshift [1] and SparkSQL [4] use Z-ordering to lay out the data; Vertica can define a sort-order over multiple columns (e.g., first age, then date), while IBM Informix, along with other spatial database systems, uses an R-Tree [15].

However, multidimensional indexes still have significant drawbacks. First, these techniques are extremely hard to tune. For example, Vertica's ability to sort hierarchically on multiple attributes requires an admin to carefully pick the sort order. The admin must therefore know which columns are accessed together, and their selectivity, to make an informed decision. Second, there is no single approach (even if tuned correctly) that dominates all others. As our experiments will show, the best multidimensional index varies depending on the data distribution and query workload. Third, most existing techniques cannot be fully tailored for a specific data distribution and query workload. While all of them provide tunable parameters (e.g., page size), they do not allow finer-grained customization for a specific dataset and filter access pattern. 
To address these shortcomings, we propose Flood, the first learned multi-dimensional in-memory index. Flood's goal is to locate records matching a query filter faster than existing indexes, by automatically co-optimizing the data layout and index structure for a particular data and query distribution.

Central to Flood are two key ideas. First, Flood uses a sample query filter workload to learn how often certain dimensions are used, which ones are used together, and which are more selective than others. Based on this information, Flood automatically customizes the entire layout to optimize query performance on the given workload. Second, Flood uses empirical CDF models to project the multi-dimensional and potentially skewed data distribution into a more uniform space. This "flattening" step helps limit the number of points that are searched and is key to achieving good performance.

Flood's learning-based approach to layout optimization distinguishes it from other multi-dimensional index structures. It allows Flood to target its performance to a particular query workload, avoid the superlinear growth in index size that plagues some indexes [9], and locate relevant records quickly without the high traversal times incurred by k-d trees and hyperoctrees, especially for larger range scans.

While Flood's techniques are general and may benefit a wide range of systems, from OLTP in-memory transaction processing systems to disk-based data warehouses, this paper focuses on improving multi-dimensional index performance (i.e., reducing unnecessary scan and filter overhead) for an inmemory column store. In-memory stores are increasingly popular due to lower RAM prices [20] and the increasing amount of main memory which can be put into a single machine $[8,19]$. In addition, Flood is optimized for reads (i.e., query speed) at the expense of writes (i.e., incremental index updates), making it most suitable for static analytical workloads, though our experiments show that adjusting to a new query workload is relatively fast. We envision that Flood could serve as the building block for a multi-dimensional in-memory key-value store or be integrated into commercial in-memory (offline) analytics accelerators like Oracle's Database In-Memory (DBIM) [35].

The ability to self-optimize allows Flood to outperform alternative state-of-the-art techniques by up to three orders of magnitude, while often having a significantly smaller storage overhead. More importantly though, Flood achieves optimality across the board: it has better, or at least on-par, performance compared to the next-fastest indexing technique on all our datasets and workloads. For example, on a real sales dataset, Flood achieves a boost of $3 \times$ over a tuned clustered column index and $72 \times$ over Amazon Redshift's Z-encoding method. On a different workload derived from TPC-H, Flood is $61 \times$ faster than the clustered column index but only $3 \times$ faster than the Z-encoding. We make the following contributions: (1) We design and implement Flood, the first learned multidimensional index, on an in-memory column store. Flood targets its layout for a particular workload by learning from a sample filter predicate distribution.

(2) We evaluate a wide range of multi-dimensional indexes on one synthetic and three real-world datasets, including one with a workload from an actual sales database at a major analytical database company. Our evaluation shows that Flood outperforms all other index structures.

(3) We show that Flood achieves query speedups on different filter predicates and data sizes, and its index creation time is competitive with existing multi-dimensional indexes.

\section{RELATED WORK}

There is a rich corpus of work dedicated to multi-dimensional indexes, and many commercial database systems have turned to multi-dimensional indexing schemes. For example, Amazon Redshift organizes points by Z-order [29], which maps multi-dimensional points onto a single dimension for sorting $[1,34,47]$. With spatial dimensions, SQL Server allows Z-ordering [27], and IBM Informix uses an R-Tree [15]. Other multi-dimensional indexes include $\mathrm{K}$-d trees, octrees, $\mathrm{R}^{*}$ trees, UB trees (which also make use of the Z-order), among many others (see [32, 41] for a survey). Flood's underlying index structure is perhaps most similar to Grid Files [31], which has many variants $[13,14,42]$. However, Grid Files do not automatically adjust to the query workload, yielding poorer performance (§7). In fact, Grid Files tend to have superlinear growth in index size even for uniformly distributed data [9].

Flood also differs from other adaptive indexing techniques such as database cracking $[16,17,38]$. The main goal of cracking is to build a query-adaptive incremental index by partitioning the data incrementally with each observed query. However, cracking produces only single dimensional clustered indexes, and does not jointly optimize the layout over multiple attributes. This limits its usefulness on queries with multi-dimensional filters. Furthermore, cracking does not take the data distribution into account and adapts only to queries; on the other hand, Flood adapts to both the queries and the underlying data.

Arguably most relevant to this work is automatic index selection [3, 25, 45]. However, these approaches mainly focus on creating secondary indexes, whereas Flood optimizes the storage and index itself for a given workload and data distribution.

For aggregation queries, data cubes [11] are an alternative to indexes. However, data cubes alone are insufficient for queries over arbitrary filter ranges, and they cannot support arbitrary actions over the queried records (e.g., returning the records themselves).

Finally, learned models have been used to replace/enhance traditional B-trees [5, 10, 23] and secondary indexes [21, 46]. Self-designing systems use learned cost models to synthesize the optimal algorithms for a data structure, resulting in 


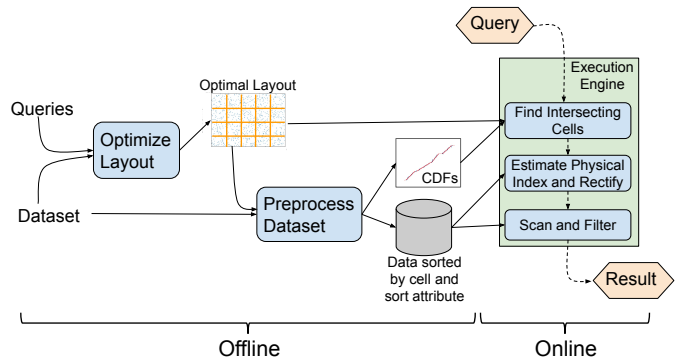

Figure 1: Flood's system architecture.

a continuum of possible designs that form a "periodic table" of data structures [18]. Flood extends these works in two ways. First, Flood learns models for indexing multiple dimensions. Since there is no natural sort order for points in many dimensions, Flood requires a design tailored specifically to multi-dimensional data. Second, prior work focused solely on constructing models of the data, without taking queries into account. Flood optimizes its layout by learning from the query workload as well. Also unlike [18], Flood embeds models into the data structure itself.

SageDB [22] proposed the idea of a learned multi-dimensional index but did not describe any details.

\section{INDEX OVERVIEW}

Flood is a multi-dimensional clustered index that speeds up the processing of relational queries that select a range over one or more attributes. For example:

SELECT SUM(R.X)

FROM MyTable

WHERE $(a \leq R . Y \leq b)$ AND $(c \leq R . Z \leq d)$

Note that equality predicates of the form $R . Z==f$ can be rewritten as $f \leq R . Z \leq f$. Typical selections generally also include disjunctions (i.e. OR clauses). However, these can be decomposed into multiple queries over disjoint attribute ranges; hence our focus on ANDs.

Flood consists of two parts: (1) an offline preprocessing step that chooses an optimal layout, creating an index based on that layout, and (2) an online component responsible for executing queries as they arrive (see Fig. 1).

At a high level, Flood is a variant of a basic grid index that divides $d$-dimensional data space into a $d$-dimensional grid of contiguous cells, so that data in each cell is stored together. We describe Flood's grid layout and online operation in $\S 3.1$ and $\S 3.2$. We then discuss Flood's central idea: how to automatically optimize the grid layout's parameters for a particular query workload ( $(4)$. The rest of this paper uses the terms attribute and dimension interchangeably, as well as the terms record and point.

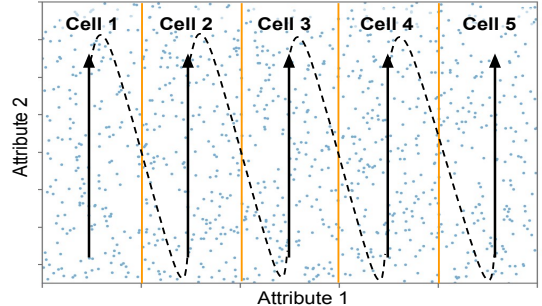

Figure 2: A basic layout in 2D, with dimension order $(\mathbf{x}, \mathbf{y})$ and $c_{0}=5$. Points are bucketed into columns along $x$ and then sorted by their $y$-values, creating the seriliaziation order indicated by the arrows.

\subsection{Data Layout}

Consider an index on $d$ dimensions. Unlike the single dimensional case, points in multiple dimensions have no natural sort order. Our first goal is then to impose an ordering over the data.

We first rank the $d$ attributes. The details of how to choose a ranking are discussed in $\S 4$, but for the purposes of illustration, we assume it is given. Next, we use the first $d-1$ dimensions in the ordering to overlay a $(d-1)$-dimensional grid on the data, where the $i$ th dimension in the ordering is divided into $c_{i}$ equally spaced columns between its minimum and maximum values. Every point maps to a particular cell in this grid, i.e. a tuple with $d-1$ attributes. In particular, if $M_{i}$ and $m_{i}$ are the maximum and minimum values of the data along the $i$ th dimension, then define the dimension's range as $r_{i}=M_{i}-m_{i}+1$. Then the cell for point $p=\left(p_{1}, \ldots, p_{d}\right)$ is:

$$
\operatorname{cell}(p)=\left(\left\lfloor\frac{p_{1}-m_{1}}{r_{1}} \cdot c_{1}\right\rfloor, \ldots,\left\lfloor\frac{p_{d-1}-m_{d-1}}{r_{d-1}} \cdot c_{d-1}\right\rfloor\right)
$$

Note that the cell is determined only by the first $d-1$ dimensions; the $d$ th dimension, the sort dimension, will be used to order points within a cell.

Flood orders the points using a depth-first traversal of the cells along the dimension ordering, i.e. cells are sorted by the first value in the tuple, then the second, etc. Within each cell, points are sorted by their value in the $d$ th dimension. Fig. 2 illustrates the sort order for a dataset with two attributes.

Flood then sorts the data by this traversal. In other words, points in cell 0 (sorted by their sort dimension) come first, followed by cell 1 , etc. Ties are broken arbitrarily.

\subsection{Basic Operation}

Flood receives as input a filter predicate consisting of ranges over one or more attributes, joined by ANDs. The intersection of these ranges defines a hyper-rectangle, and Flood's goal is to find and process exactly the points within this hyperrectangle (e.g., by aggregating them). At a high level, Flood executes the following workflow (Fig. 3): 


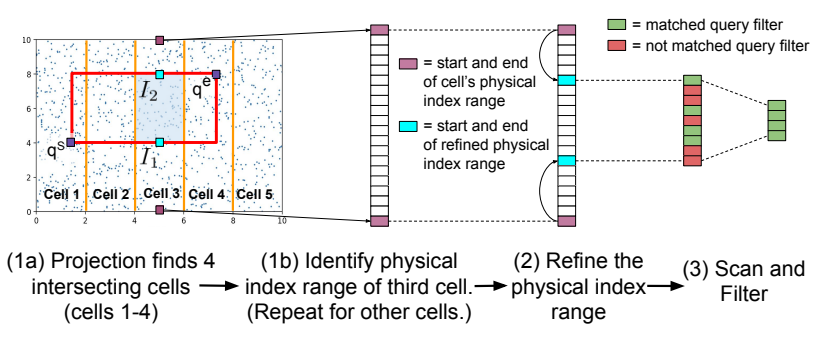

Figure 3: Basic flow of Flood's operation

(1) Projection: Identify the cells in the grid layout that intersect with the predicate's hyper-rectangle. For each such cell, identify the range of positions in storage, i.e. the physical index range, that contains that cell's points (§3.2.1).

(2) Refinement: If applicable, take advantage of the ordering of points within each cell to shorten (or refine) each physical index range that must be scanned (\$3.2.2).

(3) Scan: For each refined physical index range, scan and process the records that match the filter.

3.2.1 Projection. In order to determine which points match a filter, Flood first determines which cells contain the matching points. Since the query defines a "hyper-rectangle" in the $(d-1)$-dimensional grid, computing intersections is straightforward. Suppose that each filter in the query is a range of the form $\left[q_{i}^{s}, q_{i}^{e}\right]$ for each indexed dimension $i$. If an indexed dimension is not present in the query, we simply take the start and end points of the range to be $-\infty$ and $+\infty$, respectively. Conversely, if the query includes a dimension not in the index, that filter is ignored at this stage of query processing.

The "lower-left" corner of the hyper-rectangle is $q^{s}=$ $\left(q_{0}^{s}, \ldots, q_{d-1}^{s}\right)$ and likewise for the "upper-right" corner $q^{e}$. Both are shown in Fig. 3. Then, we define the set of intersecting cells as $\left\{C_{i} \mid \operatorname{cell}\left(q^{s}\right)_{i} \leq C_{i} \leq \operatorname{cell}\left(q^{e}\right)_{i}\right\}$. Flood keeps a cell table which records the physical index of the first point in each cell. Knowing the intersecting cells then easily translates to a set of physical index ranges to scan.

3.2.2 Refinement. When the query includes a filter over the sort dimension, Flood uses the fact that points in each cell are ordered by the sort dimension to further refine the physical index ranges to scan. In particular, suppose the query includes a filter over the sort dimension R.S of the form $a \leq R . S \leq b$. For each cell, Flood finds the physical indices of both the first point $I_{1}$ having R.S $\geq a$ and the last point $I_{2}$ such that $R . S \leq b$. This narrows the physical index range for that cell down to $\left[I_{1}, I_{2}\right]$. The simplest way to find $\left[I_{1}, I_{2}\right]$ is by performing binary search within $C$ on the values in the sort dimension. This is possible only because the points in $C$ are stored contiguously in sorted order by the sort dimension. We discuss a faster way
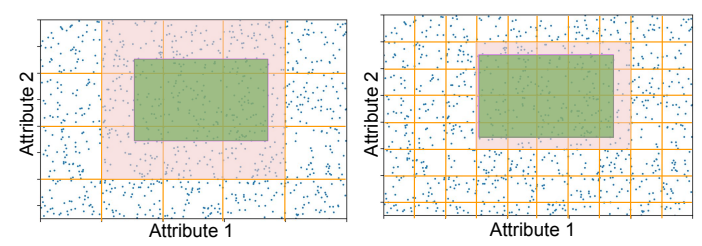

Figure 4: Doubling the number of columns can increase the number of visited cells but decreases the number of scanned points that don't match the filter (light red).

to refine, using models, in §5.2. If the query does not filter over the sort dimension, Flood skips the refinement step.

\section{OPTIMIZING THE GRID}

Flood's grid layout has several parameters that can be tuned, namely the number of columns allocated to each of the $d-1$ dimensions that form the grid, and which dimension to use as the sort dimension. Adjusting these parameters is the key way in which Flood optimizes performance on a given query workload. We found that the ordering of the $d-1$ grid dimensions did not significantly impact performance.

Adding more columns in each dimension allows Flood to scan a rectangle that more tightly bounds the true query filter, which reduces the number of points that must be scanned (Fig. 4). However, adding more columns also increases the number of sub-ranges, which incurs extra cost for projection and refinement. Striking the right balance requires choosing a layout with an optimal number of columns in each dimension.

Flood can also select the sort dimension. The sort dimension is special because it will incur no scan overhead; given a query, Flood finds the precise sub-ranges to scan in the refinement step, so that the values in the sort dimension for scanned points are guaranteed to lie in the desired range. On the other hand, the grid dimensions do incur scan overhead because a certain column might only lie partially within the query rectangle. Therefore, the choice of sort dimension can have a significant impact on performance.

It is hard to select the optimal number of columns in each dimension because it depends on many interacting factors, including the frequency of queries filtering on that dimension, the average and variance of filter selectivities on that dimension, and correlations with other dimensions in both the data and query workload. The optimal sort dimension is also hard to select for similar reasons. Therefore, we optimize layout parameters using a cost model based approach. We first describe the cost model, then present the procedure that Flood uses to optimize the layout.

\subsection{Cost Model}

Define a layout over $d$ dimensions as $L=\left(O,\left\{c_{i}\right\}_{0 \leq i<d-1}\right)$, where $O$ is an ordering of the $d$ dimensions, in which the 
$d$ th dimension is the sort dimension and $\left\{c_{i}\right\}_{0 \leq i<d-1}$ is the number of columns in the remaining $d-1$ grid dimensions.

Given a dataset $D$ and a layout $L$, we model the query time of any query $q$ as a sum of three parts, which correspond to the steps of the query flow from §3.2. Each part consists of some measurable statistic $N$, which is multiplied by a variable weight $w$ which is a function of the dataset $D$, query $q$, and layout $L$, to produce an estimate of time taken on that step:

(1) Projection contributes $w_{p} N_{c}$ to the query time, where $N_{c}$ is the number of cells that fall within the query rectangle, and $w_{p}$ is the average time to perform projection on a single cell. The weight $w_{p}$ is not constant across all datasets, queries, and layouts. For example, it is faster to identify a block of cells along a single grid dimension, which are adjacent on linear storage media, than a hypercube of cells along multiple grid dimensions which are non-adjacent.

(2) Refinement contributes $w_{r} N_{c}$ to the query time, where $w_{r}$ is the average time to perform refinement on a cell. If the query $q$ does not filter on the sort dimension, refinement is skipped and $w_{r}$ is zero. Also, $w_{r}$ is lower if the cell is smaller, because the piecewise linear CDF for that cell (explained in §5.2) is likely less complex and makes predictions more quickly.

(3) Scan contributes $w_{s} N_{s}$ to the query time, where $N_{s}$ is the number of scanned points, and $w_{s}$ is the average time to perform each scan. The weight $w_{s}$ depends on the number of dimensions filtered (fewer dimensions means fewer lookups for each scanned point), the run length of the scan (longer runs have better locality), and how many scans fall within exact sub-ranges (explained in §7.1).

Putting everything together, our model for query time is:

$$
\operatorname{Time}(D, q, L)=w_{p} N_{c}+w_{r} N_{c}+w_{s} N_{s}
$$

Given a dataset $D$ and a workload of queries $\left\{q_{i}\right\}$, we find the layout $L$ that minimizes the average of Eq. 1 for all $q \in\left\{q_{i}\right\}$.

4.1.1 Calibrating the Cost Model Weights. Since the four weight parameters $w=\left\{w_{p}, w_{r}, w_{s}\right\}$ vary based on the data, query and layout, Flood uses models to predict $w$. The features of these weight models are statistics that can be measured when running the query on a dataset with a certain layout. These statistics include $N=\left\{N_{c}, N_{s}\right\}$, the total number of cells, the average, median, and tail quantiles of the sizes of the filterable cells, the number of dimensions filtered by the query, the average number of visited points in each cell, and the number of points visited in exact sub-ranges.

As we show in $§ 7.7$, the weight models are accurate across different datasets and query workloads. In particular, when new data arrives or the query distribution changes, Flood needs only to evaluate the existing models, instead of training new ones. Flood therefore trains the weight models once to
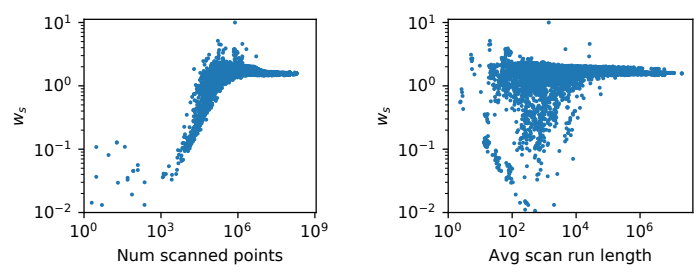

Figure 5: $w_{s}$ (and by extension, scan time) is not constant and is difficult to model analytically because of its non-linear dependence on related features.

calibrate to the underlying hardware. To produce training examples, Flood uses an arbitrary dataset and query workload, which can be synthetic. Flood generates random layouts by randomly selecting an ordering of the $d$ dimensions, then randomly selecting the number of columns in the grid dimensions to achieve a random target number of total cells. Flood then runs the query workload on each layout, and measures the weights $w$ and aforementioned statistics for each query. Each query for each random layout will produce a single training example. In our evaluation, we found that 10 random layouts produces a sufficient number of training examples to create accurate models. Flood then trains a random forest regression model to predict the weights based on the statistics.

One natural question to ask is whether a single random forest model can be trained to predict query time, instead of factoring the query time as weighted linear terms and training a model for each weight. However, a single model is inadequate because we want to accurately predict query times across a range of magnitudes; a model for query time would optimize for accuracy of slow queries at the detriment of fast queries. On the other hand, the weights span a relatively narrow range (e.g., the average time to scan a point will not vary across orders of magnitude), so are more amenable to our goal.

4.1.2 Why Use Machine Learning? We model the cost using machine learning because query time is a function of many interdependent variables with potentially non-linear relationships that are difficult to model analytically. For instance, on $10 \mathrm{k}$ training examples, Fig. 5 shows not only that the empirical average time to scan a point $\left(w_{s}\right)$ is not constant, but also that its dependence on two related features (number of scanned points and average scan run length, which affects locality) is non-linear and does not follow an obvious pattern.

Indeed, we found that query time predicted using a simple analytical model that replaces the weight parameters of Eq. 1 with fine-tuned constants has on average $9 \times$ larger difference from the true query time than our machine-learning based cost model. Furthermore, predicting the weight parameters using a linear regression model that uses the same input features as our random forest produces query time predictions with $4 \times$ larger difference from the true query time, which 
confirms that the features are interdependent and/or have non-linear relation with query time.

\subsection{Optimizing the Layout}

Given a calibrated cost model, Flood optimizes its layout for a specific dataset and query workload as follows (pseudocode is provided in Appendix B of [30]):

(1) Sample the dataset and query workload, then flatten the data sample and workload sample using RMIs trained on each dimension.

(2) Iteratively select each of the $d$ dimensions to be the sort dimension. Order the remaining $d-1$ dimensions that form the grid by the average selectivity on that dimension across all queries in the workload. This gives us $O$.

(3) For each of these $d$ possible orderings, run a gradient descent search algorithm to find the optimal number of columns $\left\{c_{i}\right\}_{0 \leq i<d-1}$ for the $d-1$ grid dimensions. The objective function is Eq. 1. For each call to the cost model, Flood computes the statistics $N=\left\{N_{c}, N_{s}\right\}$ and the input features of the weight models using the data sample instead of the full dataset $D$.

(4) Select the layout with the lowest objective function cost amongst the $d$ layouts.

Optimizing the layout is efficient (§7.7) because each iteration of gradient descent does not require building the layout, sorting the dataset, or running the query. Instead, statistics are either estimated using a sample of $D$ or computed exactly from the query rectangle and layout parameters.

\section{LEARNING FROM THE DATA}

The simple index presented in $\S 3$ does not consider or adapt to the underlying distribution of the data. Here, we present two ways that Flood learns its layout from the data. First, Flood uses a model of each attribute to better determine column spacing. Second, Flood accelerates refinement within each cell using a model of the underlying data.

\subsection{Flattening}

The index in $\S 3$ spaces columns equally, but this type of layout is inefficient when indexing highly skewed data: some grid cells will have a large number of points, causing Flood to scan too many superfluous points.

If we were to have an accurate model of each attribute's distribution, i.e. its CDF, we could choose columns such that for each attribute, each column is responsible for approximately the same number of points. In practice, Flood models each attribute using a Recursive Model Index (RMI), a hierarchy of models, e.g. linear models in our case, that is quick to evaluate [23]. The input to the model is the attribute value $v$; the output is the fraction of points with values $\leq v$. At query time, suppose that we would like to split the $k$ th dimension into $n$

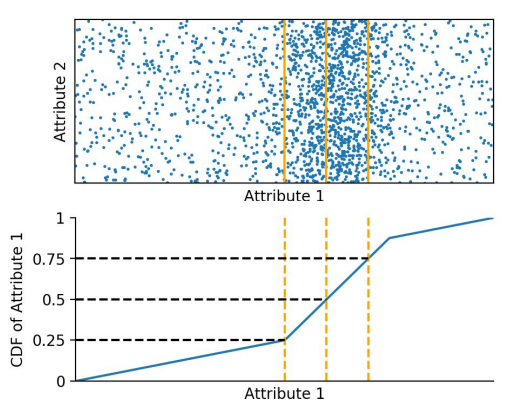

Figure 6: By flattening, each of the four columns in a dimension will contain a fourth of the points.

columns. A point with value $v$ in the $k$ th dimension will be placed into column $\lfloor\operatorname{CDF}(v) \cdot n\rfloor$. Since evaluating the RMI is efficient, we can efficiently determine the columns in each dimension that the query intersects.

Fig. 6 shows example 2-D data and the result of applying this transformation to Attribute 1. The column boundaries are no longer equally spaced across the range of Attribute 1's values. Instead, they are equally spaced in terms of the CDF of Attribute 1. This means that each of the four columns has around $1 / 4$ of the points. Since the number of points in each column is evened out, we call this a flattened layout. Flood applies this flattening transformation for each grid dimension. Skewness is abundantly present in real-world data; on two of the datasets used in our evaluation (\$7), flattening provides a performance boost of 20-30× over a non-flattened layout.

Note that while flattening may assign an equal number of points to each column of a single attribute, it does not guarantee that each cell in the final grid has a similar number of points. In particular, if two attributes are correlated, flattening each attribute independently will not yield uniformly sized cells. This may lead to some cells incurring a high scan overhead. In practice, we found that modeling single attributes, i.e. assuming each dimension is independent, was sufficient. If necessary, adding more columns per dimension can further reduce the per-cell scan overhead. Flood's layout training procedure (\$4) will choose the number of columns per dimension to trade off scan overhead with projection and refinement cost, mitigating the effect of non-uniform cell sizes. Additionally, if the correlation results in some cells being empty, those cells can be easily pruned using the cell table, incurring very little overhead. However, recent work [21, 46] suggests that it might be possible to further reduce the index size by taking advantage of the correlation. Exploring such techniques for multi-dimensional clustered indexes remains future work.

\subsection{Faster Refinement}

The simple index from §3.2.2 uses binary search over the sort dimension to refine the physical index range of each cell. In practice, since we may have to refine in every cell, binary 
search is too slow. Instead, Flood builds a CDF model over the sort dimension values for each cell. Flood uses a cell's model to estimate the endpoints of the refined physical index range, and then corrects any misprediction through a local search.

We want a model that can achieve a low average absolute error, while keeping the maximum error bounded to a reasonable value, in order for local search to be fast. Unfortunately, it is difficult to build an RMI with a target error bound. Instead, the model Flood uses is a piecewise linear model (PLM).

A PLM models a CDF by partitioning a sorted list of values $V$ into slices, each of which is modeled by a linear segment. Let $P(v)$ be the predicted index of value $v \in V$, determined by the segment responsible for the slice containing $v$, and let $D(v)$ be the index of the first occurrence of $v$. We require that the linear segments serve as a lower bound on the true CDF values, i.e. $P(v) \leq D(v)$, with the property that for every segment, the average absolute error is less than a given threshold $\delta$ :

$$
\frac{1}{|V|} \sum_{v \in V}|D(v)-P(v)| \leq \delta
$$

The lower bound property allows us to turn this condition into: $\frac{1}{|V|} \sum_{v \in V} D(v)-P(v) \leq \delta$, which is much easier to achieve.

Flood uses a greedy algorithm to partition $V$ into slices: for each $v \in V$ in increasing order, it adds $(v, D(v))$ to the segment for the current slice. If the segment's average error over the values in current slice exceeds $\delta$, it begins a new slice. The model records the smallest $v$ in each slice and forms a cache-optimized B-Tree over those values. At inference time, Flood uses the B-Tree to find the appropriate segment and thus $P(v)$. The parameter $\delta$ encodes a tradeoff between size and speed (lower $\delta$ is faster): see $\S 7.8$ for experiments tuning $\delta$ and a comparison of the PLM to other methods.

\section{DISCUSSION}

Tuning of traditional indexes. Existing multi-dimensional indexes strive for fewer hyperparameters, typically only a page size, to lower the overhead of tuning. However, fewer parameters also restricts the search space over which Flood can optimize its layout, limiting the speedups it can achieve over existing indexes. Indeed, Fig. 8 demonstrates that simply tuning page size does not offer substantial performance improvement. In contrast, Flood's grid layout intentionally offers a larger number of parameters over which to optimize, all of which can be automatically tuned by Flood's learning procedure. As a result, Flood can customize the layout for a particular query workload better than existing indexes.

Alternatives to grids. Flood is a learning-enhanced version of a basic grid index, but many alternative techniques to divide a multi-dimensional space exist (R-Tree, $\mathrm{k}$-d tree, $\mathrm{Z}$-order, etc.). We decided to use a grid structure for several reasons. First, it has a small space overhead: other multi-dimensional indexes use between 10MB and 1GB, but Flood's grid uses less than $1 \mathrm{kB}(\$ 7)$, leaving ample room to add per-cell models. Second, the grid has low lookup latency, since it avoids pointer chasing. On the TPC-H dataset in §7, Flood with flattening takes $0.46 \mathrm{~ms}$ to identify relevant grid cells (excluding refinement), while the $\mathrm{k}-\mathrm{d}$ tree and hyperoctree take $8.9 \mathrm{~ms}$ $(20 \times)$ and $1.8 \mathrm{~ms}(4 \times)$ to identify matching pages, respectively. This trend is consistent across the datasets we evaluate on. Z-order based indexes have low lookup times but expose no obvious parameters that can be tuned for the query workload. Note that our flattening approach is necessary to keep scan times low by making sure the grid is not highly imbalanced.

Nearest Neighbor Queries. Tree-based indexes that are used for geospatial data, such as k-d trees and R-trees, support $k$-nearest neighbor $(\mathrm{kNN})$ queries. For example, a k-d tree locates the page with the query point and checks adjacent pages until all $k$ neighbors are found. Flood can easily locate adjacent cells in its grid layout, allowing a similar kNN algorithm. However, since this paper does not focus on geospatial analytics, we exclude kNN queries from our evaluation.

Multi-dimensional CDFs. In $§ 5.1$, we mentioned that correlated dimensions can yield non-uniform data after flattening. To address this issue, for each pair of correlated dimensions, one could train a 2-dimensional joint CDF, or train a conditional CDF that creates a 1-D model for attribute $A$ within each column of attribute B. However, it is difficult to ensure that a multi-dimensional RMI model gives monotonic predictions along each dimension, which is a necessary property for partitioning points into columns; and conditional CDFs did not significantly improve performance in our benchmarks, but did significantly increase index size. Therefore, Flood does not use multi-dimensional CDFs. Efficiently modeling correlations between more dimensions is an active area of research [36, 44].

\section{EVALUATION}

We first describe the experimental setup and then present the results of an in-depth experimental study that compares Flood with several other indexing methods on a variety of datasets and workloads. Overall, this evaluation shows that:

(1) Flood achieves optimality across the board: it is faster than, or on par with, every other index on the tested workloads. However, the next best index changes depending on the dataset. On our datasets, Flood is up to $187 \times$ faster than a single-dimensional clustered column index, up to $62 \times$ faster than a Grid File, up to $72 \times$ faster than a Z-order index, up to $250 \times$ faster than an UB-tree, up to $43 \times$ faster than a hyperoctree, and up to $48 \times$ faster than a k-d tree or R-tree.

(2) Flood's index can take up to $50 \times$ less space than the next fastest index.

(3) Even though we did not optimize Flood for dynamic workloads, Flood can train its layout and reorganize the records 
quickly for a new query distribution, typically in under a minute for a 300 million record dataset.

(4) Flood's performance over baseline indexes improves with larger datasets and higher selectivity queries.

\subsection{Implementation}

We implement Flood in $\mathrm{C}++$ on a custom column store that uses block-delta compression: in each column, the data is divided into consecutive blocks of 128 values, and each value is encoded as the delta to the minimum value in its block. Our encoding scheme allows constant-time element access and is able to compress the datasets used in our evaluation by $77 \%$.

Our implementation uses 64-bit integer-valued attributes. Any string values are dictionary encoded prior to evaluation. Floating point values are typically limited to a fixed number of decimal points (e.g., 2 for price values). We scale all values by the smallest power of 10 that converts them to integers. We include two scan-time optimizations:

(1) If the range of data being scanned is exact, i.e., we are guaranteed ahead of time that all elements within the range match the query filter, we skip checking each value against the query filter. For common aggregations, e.g. COUNT, this removes unnecessary accesses to the underlying data.

(2) Similar to the idea of [24], our implementation allows indexes to speed up common aggregations like SuM by including a column in which the $i$ th value is the cumulative aggregation of all elements up to index $i$. In the case of an exact range, the final aggregation result is simply the difference between the cumulative aggregations at the range endpoints. Note that this is not a data cube as we can support arbitrary ranges instead of only pre-aggregated ranges.

These additions are meant to demonstrate that Flood can take advantage of features that existing indexes enjoy. We show in $§ 7.5$ that these optimizations are not required for Flood: its performance benefits are due primarily to the optimality of the layout and not the details of the underlying implementation. Our random forest regression uses Python's Scipy library [39].

We benchmark our column store with MonetDB, an opensource column store [28], by executing a query workload with full scans. Both MonetDB and our implementation were run single-threaded, with identical bit widths for each attribute, and without compression. Note that MonetDB does not support compression on numerical columns. Averaged over 150 aggregation queries on the TPC-H dataset (§7.3), our scan times are within $5 \%$ of MonetDB, showing that our column store implementation is on par with existing systems.

\subsection{Baselines}

We compare Flood to other solutions implemented on the same column store, with the same optimizations, if applicable: (1) Full Scan: Every point is visited, but only the columns present in the query filter are accessed.
(2) Clustered Single-Dimensional Index: Points are sorted by the most selective dimension in the query workload, and we learn a B-Tree over this sorted column using an RMI [23]. If a query filter contains this dimension, we locate the endpoints using the RMI. Otherwise, we perform a full scan. Since a clustered index spends a vast majority of its time scanning instead of indexing, an RMI-based approach performs comparably to a standard B-tree: their total query times are within $1 \%$ of each other. Therefore, we show results only for the RMI-based index.

(3) Grid Files [31] index points by assigning them to cells in a grid, similar to Flood. However, unlike Flood, Grid File columns are determined incrementally and do not optimize for a query workload. Additionally, points in multiple adjacent cells may be stored together in the same bucket and are not sorted. We found that the Grid Files algorithm in [31] requires a long time to construct on heavily skewed data, so we omit results when it took over an hour.

(4) The Z-Order Index is a multidimensional index that orders points by their $Z$-value [9]; contiguous chunks are grouped into pages. Given a query, the index finds the smallest and largest $\mathrm{Z}$-value contained in the query rectangle and iterates through each page with $Z$-values in this range.

(5) The UB-tree [37] also indexes points using their Z-values. A query finds the range of points to scan in the same manner as the Z-Order Index. The UB-tree has the ability to skip forward to the next $Z$-value contained in the query rectangle, which avoids unnecessary scans.

(6) The Hyperoctree [26] recursively subdivides space equally into hyperoctants (the $d$-dimensional analog to 2-dimensional quadrants), until the number of points in each leaf is below a predefined but tunable page size.

(7) The $k$ - $d$ tree recursively partitions space using the median value along each dimension, until the number of points in each leaf falls below the page size. The dimensions are selected in a round robin fashion, in order of selectivity.

(8) The $R^{*}$-Tree is a read-optimized variant of the $\mathrm{R}$-Tree that is bulk loaded to optimize for read query performance. We benchmark the $\mathrm{R}^{*}$-Tree implementation from libspatialindex [12]. On larger datasets, the $\mathrm{R}^{*}$-Tree was prone to outof-memory errors and was not included in benchmarks.

While some techniques are read-optimized like Flood (e.g., the Z-Order and $R^{*}$-Tree), others are inherently more writefriendly (e.g., UB-tree); we still include them for the sake of comparison while optimizing them for reads as much as possible (e.g., using dense cache-aligned pages). Additional implementation details can be found in Appendix A of [30].

Our primary goal is to evaluate the performance of our multidimensional index as a fundamental building block for improving range request with predicates (e.g., filters) over one or more attributes. We therefore do not evaluate against other full-fledged database systems or queries with joins, group-bys, 


\begin{tabular}{lllll}
\hline & sales & tpc-h & osm & perfmon \\
\hline records & $30 \mathrm{M}$ & $300 \mathrm{M}$ & $105 \mathrm{M}$ & $230 \mathrm{M}$ \\
queries & 1000 & 700 & 1000 & 800 \\
dimensions & 6 & 7 & 6 & 6 \\
size (GB) & 1.44 & 16.8 & 5.04 & 11
\end{tabular}

Table 1: Dataset and query characteristics.

or other complex query operators. While the impact of our multi-dimensional clustered index on a full query workload for an in-memory column-store database system would be interesting, it requires major changes to any available opensource column-store and is beyond the scope of this paper. However, it should be noted that that even in its current form, Flood could be directly used as a component to build useful services, such as a multi-dimensional key-value store.

For a fair comparison, all benchmarks use a single thread without SIMD instructions. We exclude multiple threads mainly because our baselines were not optimized for it. We discuss how Flood could take advantage of parallelism and concurrency in §8. All experiments are run on an Ubuntu Linux machine with an Intel Core i9 3.6GHz CPU and 64GB RAM.

\subsection{Datasets}

We evaluate indexes on three real-world and one synthetic dataset, summarized in Tab. 1. Queries are either real workloads or synthesized for each dataset, and include a mix of range filters and equality filters. The Sales dataset is a 6attribute dataset and corresponding query workload drawn directly from a sales database at a commercial technology company. It was donated to us by a large corporation on the condition of anonymity. The dataset consists of 30 million records, with an anonymizing transformation applied to each dimension. Each query in this workload was submitted by an analyst as part of report generation and analysis at the corporation.

Our second real-world dataset, OSM, consists of all 105 million records catalogued by the OpenStreetMap [33] project in the US Northeast. All elements contain 6 attributes, including an ID and timestamp, and $90 \%$ of the records contain GPS coordinates. Our queries answer relevant analytics questions, such as "How many nodes were added to the database in a particular time interval?" and "How many buildings are in a given lat-lon rectangle?" Queries use between 1 and 3 dimensions, with range filters on timestamp, latitude, and longtitude, and equality filters on type of record and landmark category. Each query is scaled so that the average selectivity is $0.1 \% \pm 0.013 \%$.

The performance monitoring dataset Perfmon contains logs of all machines managed by a major US university over the course of a year. Our queries include filters over time, machine name, CPU usage, memory usage, swap usage, and load average. The data in each dimension is non-uniform and often highly skewed. The original dataset has $23 \mathrm{M}$ records, but we use a scaled dataset with $230 \mathrm{M}$ records.

Our last dataset is TPC-H [43]. For our evaluation, we use only the fact table, linei tem, with $300 \mathrm{M}$ records (scale factor 50) and create queries by using filters commonly found in the TPC-H query workload, with filter ranges scaled so that the average query selectivity is $0.1 \%$. Our queries include filters over ship date, receipt date, quantity, discount, order key, and supplier key, and either perform a SUM or COUNT aggregation.

For each dataset, we generate a train and test query workload from the same distribution. Flood's layout is optimized on the training set, and we only report results on the test set.

\subsection{Results}

Overall Performance. We first benchmark how well Flood can optimize for a query workload compared to baseline indexes that are also optimized for the same query workload. Fig. 7 shows the query time for each optimized index on each dataset. Flood uses the layout learned using the algorithm in $\S 4$, while we tuned the baseline approaches as much as possible per workload (e.g., ordered dimensions by selectivity and tuned the page sizes). This represents the best case scenario for the other indexes: that the database administrator had the time and ability to tune the index parameters.

On three of the datasets, Flood achieves between $2.4 \times$ and $3.3 \times$ speedup on query time compared to the next closest index, and is always at least on-par, thus achieving the best performance across-the-board. However, the next best system changes across datasets. Thus, depending on the dataset and workload, Flood can outperform each baseline by orders of magnitude. For example, on the real-world sales dataset, Flood is at least $43 \times$ faster than each multi-dimensional index, but only $3 \times$ faster than a clustered index. However, on the TPC-H dataset, Flood is $187 \times$ faster than a clustered index.

On every dataset, Fig. 8 shows that Flood beats the Pareto frontier set by the other multi-dimensional indexes. In particular, even though Flood's performance on OSM is on par with the hyperoctree, its index size is more than $20 \times$ smaller. The hyperoctree thus has to spend much more memory for its performance than Flood. Flood's space overhead comes partially from the grid layout metadata, but mostly (over 95\%) from the models of the sort attribute it maintains per cell.

Different Workload Characteristics. In practical settings, it is unlikely that a database administrator will be able to manually tune the index for every workload change. The ability of Flood to automatically configure its index for the current query workload is thus a significant advantage. We measure this advantage by tuning all indexes for the workloads in Fig. 7, and then changing the query workload characteristics to:

(1) Single record filters, i.e. point lookups, using one or two ID attributes, as commonly found in OLTP systems. 

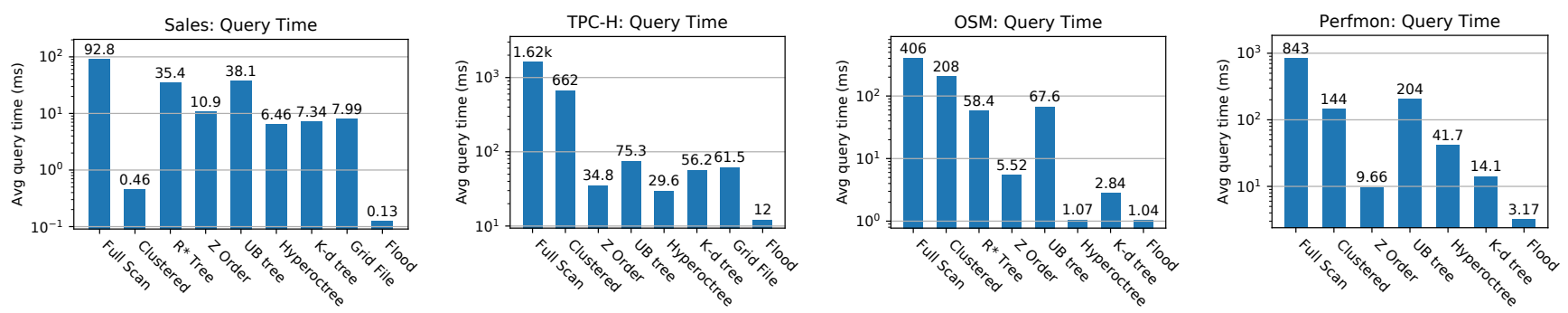

Figure 7: Query latency of Flood on all datasets. Flood's index is trained automatically, while other indexes are manually tuned for optimal performance on each workload. We exclude the $\mathbf{R}^{*}$-tree when it ran out of memory. Note the $\log$ scale.

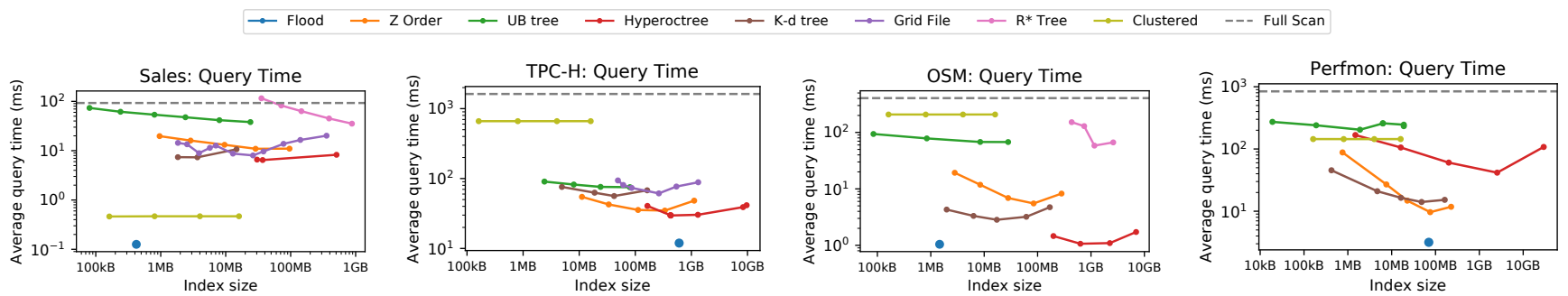

Figure 8: Flood (blue) sees faster performance with a smaller index, pushing the pareto frontier. Note the log scale.
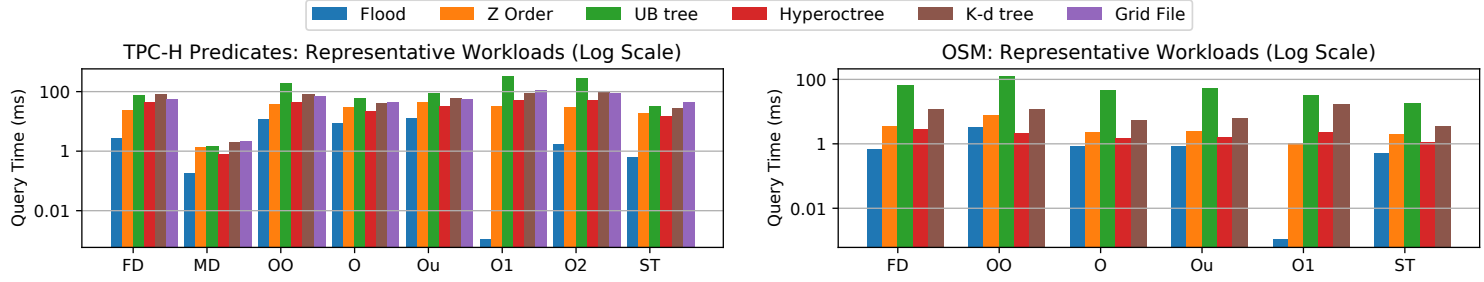

Figure 9: Flood and other indexes on workloads that have: fewer dimensions than the index (FD), as many dimensions as the index (MD), a skewed OLAP workload (O), a uniform OLAP workload (Ou), an OLTP workload over a single primary key (i.e., point lookups) (O1) and two keys (O2), a mixed OLTP + OLAP workload (OO), and a single query type (ST). Note the log scale.

(2) An OLAP workload, similar to the ones in Fig. 7, that answer reasonable business questions about the underlying dataset. Some types of queries occur more often than others, skewing the workload.

(3) An OLAP workload where each query type is equally likely.

(4) An equal split of workloads (1) and (2), i.e., combined OLTP and OLAP queries.

(5) A workload with a single type of query, using the same dimensions with the same selectivities.

(6) A workload with fewer dimensions (a strict subset) than indexed by the baseline indexes.

Fig. 9 shows the potential advantages Flood can achieve over more static alternatives. Flood consistently beats other indexes, though the magnitude of improvement depends on the dataset and query workload. For example, on TPC-H, Flood achieves a speedup of more than $20 \times$ on half the workloads, while on OSM, the median improvement is $2.2 \times$.

Dynamic Query Workload Changes. Here, we demonstrate how the performance of Flood varies over several random workloads, when the administrator does not tune the other indexes. We created 30 random workloads for the TPC$\mathrm{H}$ dataset. Each workload runs for one hour and consists of at most 10 distinct query types, and each query type in turn consists of up to 6 dimensions, both chosen uniformly at random. The selectivities of each dimension are chosen randomly, with the constraint that all queries have an average total selectivity of around $0.1 \%$ and are more selective on key attributes.

Fig. 10 shows the results over time with Flood being the only index that changes from one hour to the next (all others were kept fixed and tuned for the workload in Fig. 7). At the start of each hour, a new query workload is introduced, and 


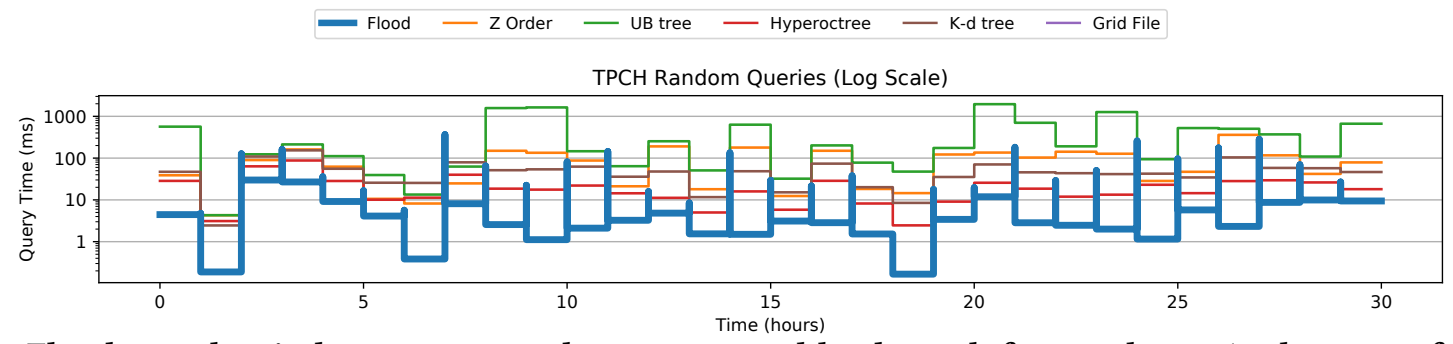

Figure 10: Flood vs. other indexes on 30 random query workloads, each for one hour. At the start of each hour, Flood's performance degrades, since it is not trained for the new workload; however, it recovers in 5 minutes on average once the layout is re-learned, and beats the next best index by $5 \times$ at the median. Note the $\log$ scale.

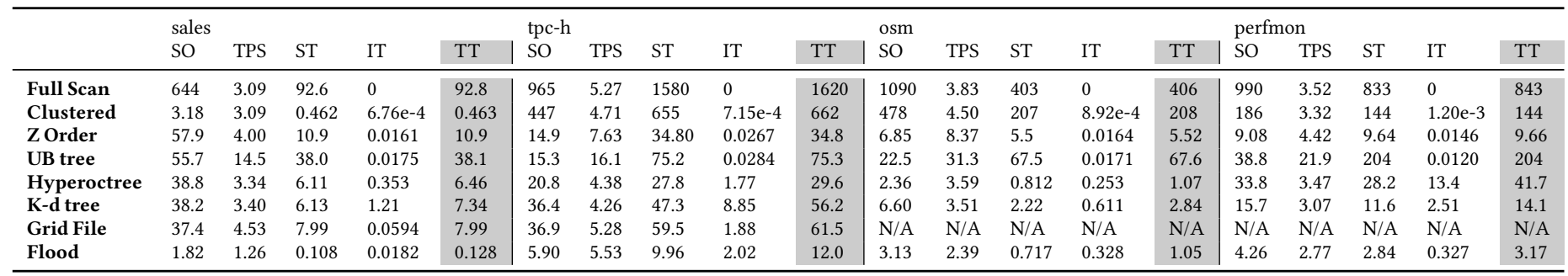

Table 2: Performance breakdown: scan overhead (SO), i.e. the ratio between points scanned and result size; average time spent scanning per scanned point, in nanoseconds (TPS); average time spent scanning, in milliseconds (ST); average time spent indexing (for Flood this includes projection and refinement), in milliseconds (IT); total query time, in milliseconds (TT). SO $\times$ TPS is proportional to ST, and $\mathrm{ST}+\mathrm{IT}+\epsilon=\mathrm{TT}$ (a small fraction of query time is spent neither scanning nor indexing). $R^{*}$-tree omitted because instrumentation for collecting statistics was inadequate in [12].
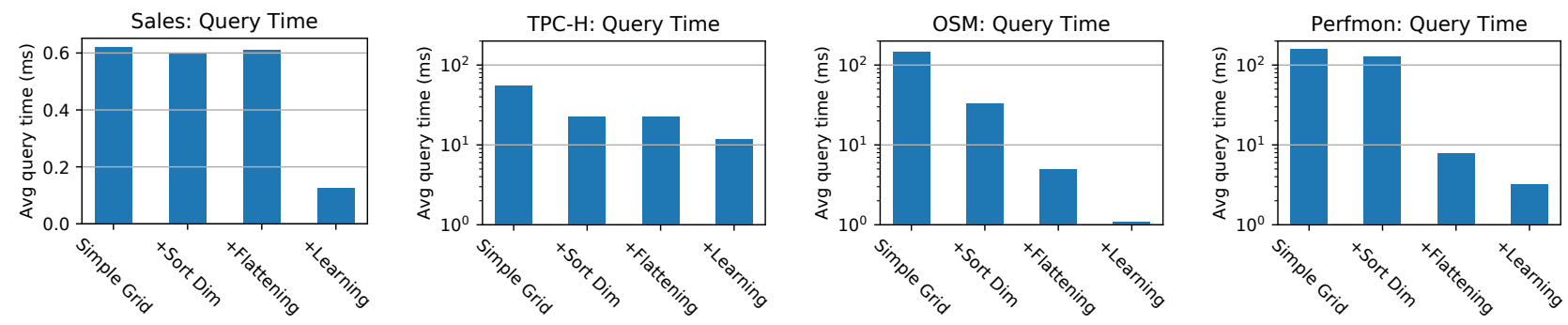

Figure 11: Flattening and learning help Flood achieve low query times but is workload dependent.

we trigger Flood's retraining. During the retraining phase, which we assume happens on a separate instance, Flood runs the new queries on its old layout, causing brief performance degradation and producing a spike at the start of each hour. It only switches to the new, more performant layout once retraining is finished. Flood outperforms all other indexes, showing a median improvement of more than $5 \times$ over the closest competitor, with $30 \%$ of queries achieving more than a $10 \times$ speedup. The results suggest that Flood is able to generalize well by adapting to new and unforeseen workloads.

Fig. 10 also highlights the importance of learning from the query workload. When transitioning to the next query workload, Flood's performance often worsens, since the current layout is usually not suitable for the new workload. Re-learning the layout based on the new workload lowers query time back lower than other indexes. Learning a layout is therefore (a) effective at adapting to new query workloads and (b) crucial to Flood's performance improvement over other indexes. We leave the detection of workload changes to future work (\$8).

While the results are encouraging, it is also important to consider the time it takes to adjust to a new query workload. Flood takes at most around 1 minute to adapt to a new query workload, but it more than makes up for this adjustment period through improved performance on the subsequent workload. We evaluate index creation time in further detail in $§ 7.7$.

Performance Breakdown. Where does Flood's advantage over baseline indexes come from? We look at the scan overhead, the ratio of total points scanned by the index to points matching the query. The scan overhead is implementation agnostic: it relies neither on the machine nor on the implementation of the underlying column store. A high scan overhead suggests that the index wastes time scanning unnecessary points. Since all indexes spend the vast majority of their time scanning, the scan overhead is a good proxy for overall query performance. 

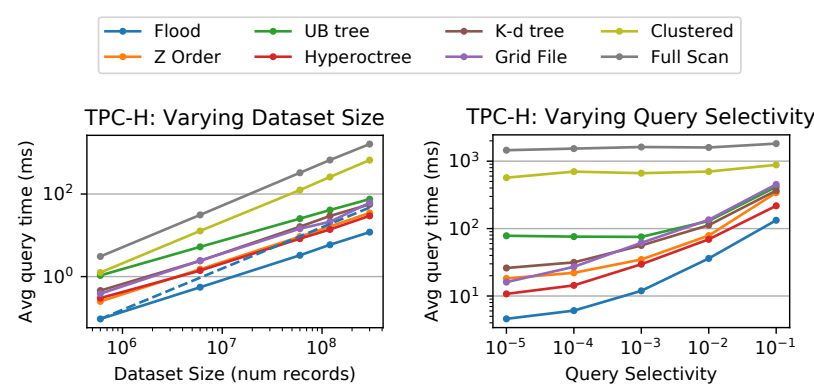

Figure 12: Flood's performance scales both with dataset size and query selectivity. The dashed blue line depicts what linear scaling would like like.

Tab. 2 shows that Flood achieves the lowest scan overhead (SO) on three out of four datasets, which confirms that Flood's optimized layout is able to better isolate records that match a query filter. Additionally, Flood usually spends less time per scanned point (TPS) because Flood avoids accessing the sort dimension. As a result, Flood consistently achieves the lowest scan time (ST), which is proportional to the product of $\mathrm{SO}$ and TPS. This more than makes up for Flood's higher index time (IT), which includes the time to project and refine. Indexes based on Z-order must compute $\mathrm{Z}$-values and thus have a higher time per scanned point. Tree-based indexes have the highest index time due to the overhead of tree traversal.

Which of Flood's components is responsible for its performance? Fig. 11 shows the incremental benefit of (1) sorting the last indexed dimension instead of creating a $d$-dimensional histogram, (2) flattening the data instead of using columns of fixed width, and (3) adapting to the query workload using the training procedure from $\S 4$. The baseline system is a "Simple Grid" on all $d$ dimensions, with the number of columns in each dimension proportional to that dimension's selectivity. (1) offers marginal benefits: it allows more columns to be allocated to the first $d-1$ dimensions, increasing the resolution of the index along those dimensions without increasing the total number of cells. The biggest improvements come from (2) and (3). However, the effect of each varies across datasets. Flattening benefits OSM and Perfmon since both datasets have heavily skewed attributes. Since Sales and TPC-H data are fairly uniform, using a non-flattened layout performs equally well. Finally, learning from queries provides major performance gains on all datasets, corroborating results from Fig. 10.

\subsection{Scalability}

Dataset Size. To show how Flood scales with dataset size, we sample records from the TPCH dataset to create smaller datasets. We train and evaluate these smaller datasets with the same train and test workloads as the full dataset. Fig. 12a shows that the query time of Flood grows sub-linearly. As the number of records grows, the layout learned by Flood

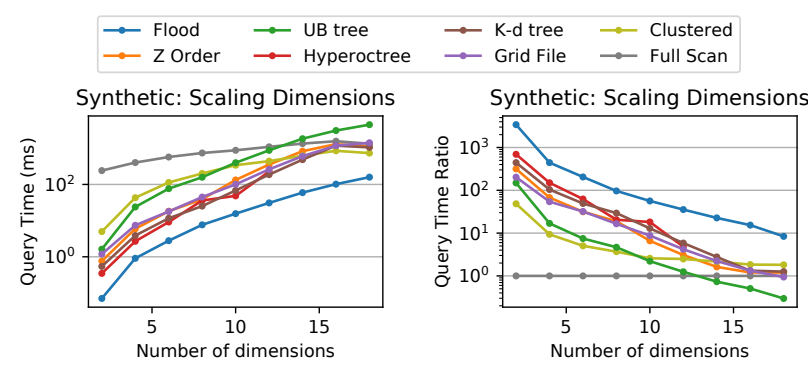

Figure 13: Query time, both (a) absolute and (b) relative to a full scan, as the number of dimensions varies.

uses more columns in each dimension, which results in more cells. The extra overhead incurred by processing more cells is outweighed by the benefit of lowering scan overhead.

Query Selectivity. To show how Flood performs at different query selectivities, we scale the filter ranges of the queries in the original TPC-H workloads up and down equally in each dimension in order to achieve between $0.001 \%$ and $10 \%$ selectivity. Fig. 12b shows that Flood performs well at all selectivities. The performance benefit of Flood is less apparent at $10 \%$ selectivity because all indexes are able to incur lower scan overhead when more points fall into the query rectangle.

Number of Dimensions. To show how Flood scales with dimensions, we create synthetic $d$-dimensional datasets $(d \leq 18)$ of 100 million records with each dimension sampled from i.i.d. uniform distributions. For each dataset, we create a query workload of 1000 queries. The number of dimensions filtered in the queries varies uniformly from 1 to $d$. If a query has a filter on $k$ dimensions, they are the first $k$ dimensions in the dataset. For each query, the filter selectivity along each dimension is the same and is set so that the overall selectivity is $0.1 \%$. For example, for a 2 -dimensional dataset, 500 queries will select $0.1 \%$ of the domain of dimension 1 , and 500 queries will select around $3.2 \%$ of the domains of dimensions 1 and 2 .

Fig. 13a shows that Flood continues to outperform the baseline indexes at higher dimensions. Note that the clustered index's relative performance also improves, since the baseline indexes spend resources on dimensions which are not frequently filtered on. By contrast, Flood learns which dimensions to prioritize and excludes the least frequently filtered dimensions from the index on higher-dimensional datasets. Yet, Flood is also impacted by the curse of dimensionality (Fig. 13b), which depicts the speedup of each index compared to a full scan. However, Flood can dampen this effect through its selfoptimization, degrading more slowly than other indexes.

\subsection{The Cost Model}

Finding the Optimum. Choosing an optimal layout requires balancing two competing factors: reducing the latency of locating both the relevant cells and physical index ranges within 

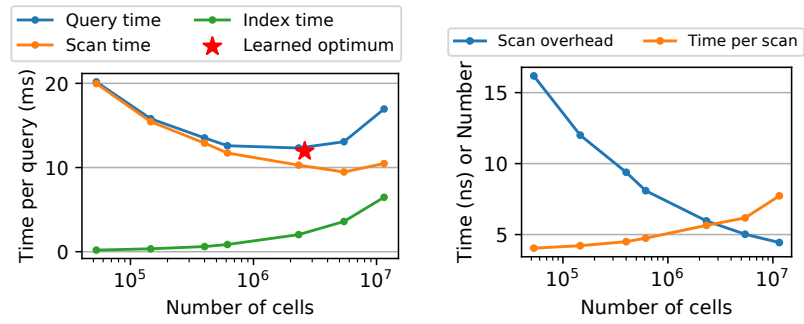

Figure 14: TPC-H: Adding cells reduces scan overhead but incurs a higher indexing cost and worse locality.

\begin{tabular}{|c|c|c|c|c|c|}
\hline & & $\begin{array}{l}\text { Layout lear } \\
\text { sales }\end{array}$ & $\begin{array}{l}\text { ned for } \\
\text { tpc-h }\end{array}$ & osm & perfmon \\
\hline \multirow{4}{*}{ 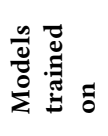 } & sales & 0.128 & $10.8(-8 \%)$ & $0.975(-7 \%)$ & $3.49(+17 \%)$ \\
\hline & tpch & $0.132(+3 \%)$ & 11.7 & $0.986(-6 \%)$ & $3.18(+6 \%)$ \\
\hline & osm & $0.134(+5 \%)$ & $11.7(+0 \%)$ & 1.05 & $3.14(+5 \%)$ \\
\hline & perfmon & $0.137(+7 \%)$ & $11.6(-1 \%)$ & $0.964(-8 \%)$ & 2.99 \\
\hline
\end{tabular}

Table 3: Query time (ms) when layouts are learned using cost models trained on different examples.

each cell (index time), and reducing the scan time by lowering scan overhead. Fig. 14a illustrates this trade-off as the grid size changes (we fix a layout and scale the number of columns in each dimension proportionally): as the number of cells grows, scan time decreases because scan overhead decreases, but index time increases because there are more cells to process.

Fig. 14a shows that Flood finds the number of cells that minimizes the total query time (red star), i.e., the best tradeoff between scan time and index time. Note that we show the cost surface along only a single axis for visual clarity.

Robustness of the model. As per §4.1.1, our cost model needs to learn the weights $\left\{w_{p}, w_{l}, w_{r}, w_{s}\right\}$. This calibration step should happen once per dataset and machine. On our server, it took around 10 minutes, most of which was spent generating training examples. However, maybe surprisingly, the weights are robust to the data itself, so the cost model does not need to be retrained for every dataset. To show this, we trained our cost model on each of our four datasets, used each model to learn layouts for all four datasets, and then ran all 16 layouts on the corresponding query workloads. Tab. 3 shows that, no matter which dataset is used to learn the layout, the resulting layouts have similar performances, often with less than a $10 \%$ difference between them. Therefore, Flood can use the same cost model regardless of changes to the dataset or query workload. This makes calibration a one-time cost of 10 minutes.

\subsection{Index Creation}

Tab. 4 shows the time to create each index. We separate index creation time for Flood into learning time, which is the time taken to learn the layout ( $\$ 4.2)$; and loading time, which is the time to build the primary index. The reported learning times use sampling of the dataset and query workload, described

\begin{tabular}{lllll}
\hline & sales & tpc-h & osm & perfmon \\
\hline Flood Learning & 10.3 & 33.4 & 44.5 & 33.3 \\
Flood Loading & 4.12 & 29.6 & 8.03 & 22.0 \\
Flood Total & 14.4 & 63.0 & 52.5 & 55.3 \\
\hline Clustered & 2.11 & 16.2 & 4.85 & 11.6 \\
Z Order & 7.82 & 86.7 & 24.9 & 72.6 \\
UB tree & 8.28 & 81.9 & 26.0 & 69.5 \\
Hyperoctree & 2.47 & 42.2 & 31.4 & 54.8 \\
K-d tree & 8.45 & 140 & 36.9 & 250 \\
Grid File & 10.6 & 121 & N/A & N/A \\
R $^{*}$ tree & 259 & N/A & 1340 & N/A \\
\hline
\end{tabular}

Table 4: Index Creation Time in Seconds

next. The total index creation time of Flood is competitive with the creation time of the baseline indexes.

Sampling records. Optimizing the layout using the entire dataset and query workload can take prohibitively long and does not scale well. However, Flood can reduce learning time without a significant performance loss by sampling the data. Fig. 15 shows that Flood maintains low query times when estimating features with a $0.01-1 \%$ sample. This is because the main purpose of the sample is to estimate the number of records scanned per query. With selectivities around $0.1 \%$ or higher, a sample of $1 \%$ records is sufficient. Yet, this alone is not enough to match the creation time of the hyperoctree, the fastest of our multi-dimensional baselines.

Sampling queries. Sampling the query workload can further reduce Flood's creation time. Here we conservatively use a data sample size of $100 \mathrm{k}$ records and vary the query sample size. As Fig. 16 shows, Flood maintains low query times when using only $5 \%$ of queries. This is because the query workloads contain limited number of query types. Since queries within each type have similar characteristics with respect to selectivity and which dimensions are filtered, Flood only requires a few queries of each type to learn a good layout. However, the variance in performance increases as the query workload sample size decreases. With both optimizations (data and query samples), Flood achieves a learning time on par with the hyperoctree creation time without sacrificing performance.

\subsection{Per-cell Models}

$\$ 5.2$ discusses CDF models to accelerate refinement. Since these CDFs are evaluated twice for each visited cell (beginning and end of the range), small speedups in lookup time may be noticeable on overall query time. Fig. 17a benchmarks the lookup time, including inference and an exponential search rectification phase, of three options we considered: the PLM (our approach), the learned B-tree [23], and binary search. We used real data (timestamps from the OSM dataset) and synthetic staggered uniform data (uniform over identically sized but disjoint intervals), with query points sampled from 

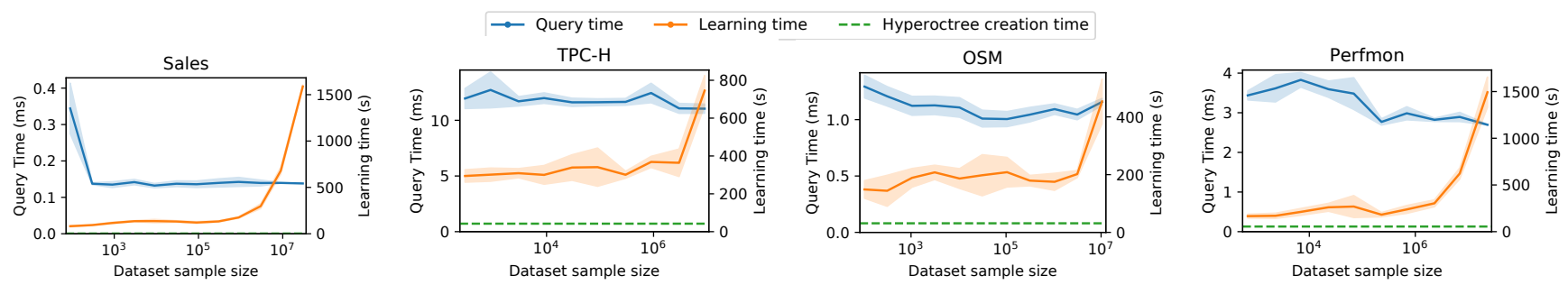

Figure 15: Learning time and resulting query time when sampling the dataset over several trials. One standard deviation from the mean is shaded. For comparison, we show the index creation time for the hyperoctree.
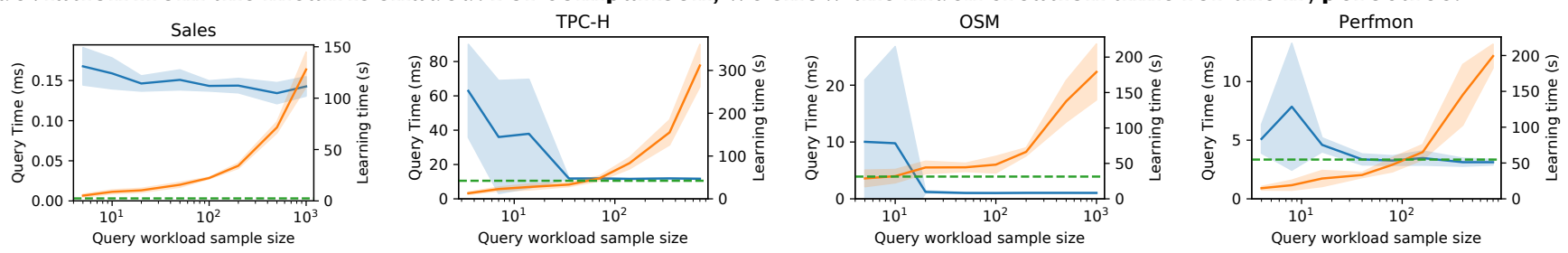

Figure 16: Learning time and resulting query time when sampling the queries over several trials. One standard deviation from the mean is shaded. For comparison, we show the index creation time for the hyperoctree.
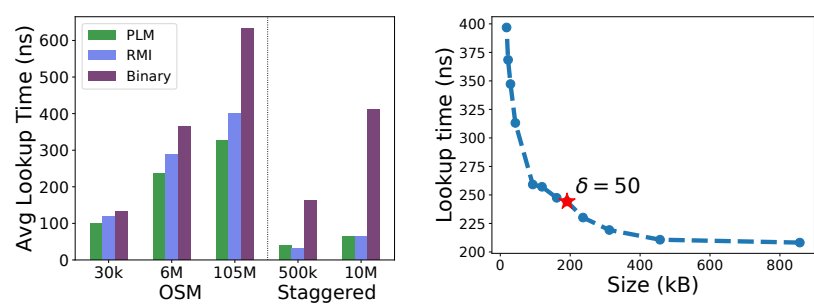

Figure 17: (a) Three per-cell CDF models on two 1-D datasets. (b) The PLM's size-speed tradeoff, with our configuration marked.

each dataset. The PLM and RMI perform comparably, and both beat binary search by up to $4 \times$. We use the PLM since it requires only a single tuning parameter $\delta$, which encodes the tradeoff between accuracy and size (Fig. 17b). By contrast, the learned B-tree [23] requires extensive tuning of the number of experts per layer. The choice of $\delta$ depends on how much the administrator prioritizes speed over space: Fig. 17b shows that $\delta=50$ strikes a reasonable balance.

\section{FUTURE WORK}

Shifting workloads. Flood can quickly adapt to workload changes but cannot detect when the query distribution has changed sufficiently to merit a new layout. To do this, Flood could periodically evaluate the cost $(\S 4)$ of the current layout on queries over a recent time window. If the cost exceeds a threshold, Flood can replace the layout.

Additionally, Flood is completely rebuilt for each new workload. However, Flood could also be incrementally adjusted, e.g. by coalescing adjacent columns or splitting a column, or by incorporating aspects of incremental layout creation from database cracking [16], to avoid rebuilding the index.
Insertions. Flood currently only supports read-only workloads. To support insertions, each cell could maintain gaps, similar to the fill factor of a B+Tree. It could also maintain a delta index [40] in which updates are buffered and periodically merged into the data store, similar to Bigtable [2].

Concurrency and parallelism. Flood is currently singlethreaded, but it can be extended to take advantage of concurrency and parallelism. Different cells can be refined and scanned simultaneously; within a cell, records can be scanned in parallel, allowing Flood to benefit from multithreading. Additionally, since Flood stores each column in the column store as a dense array, it can also take advantage of SIMD.

\section{CONCLUSION}

Despite the shift of OLAP workloads towards in-memory databases, state-of-the-art systems fail to take advantage of multi-dimensional indexes to accelerate their queries. Many instead opt for simple 1-D clustered indexes with bulky secondary indexes. We design Flood, a new multi-dimensional primary index that is jointly optimized using both the underlying data and query workloads. Learning from the query workload allows Flood to beat existing approaches: Flood outperforms optimally tuned state-of-the-art approaches by $30-$ $400 \times$, while using a fraction of the space. Our results suggest that learned primary multi-dimensional indexes can serve as useful building blocks in larger in-memory database systems.

Acknowledgements. This research is funded by Google, Intel, and Microsoft as part of the MIT Data Systems and AI Lab (DSAIL), by NSF Awards IIS-1900933 and CNS-1751009, by DARPA Award 16-43-D3M-FP040, and by a Microsoft Research Faculty Fellowship. 


\section{REFERENCES}

[1] Amazon AWS. 2016. Amazon Redshift Engineering's Advanced Table Design Playbook: Compound and Interleaved Sort Keys. https://aws.amazon.com/blogs/big-data/amazon-redshiftengineerings-advanced-table-design-playbook-compound-andinterleaved-sort-keys/.

[2] Fay Chang, Jeffrey Dean, Sanjay Ghemawat, Wilson C. Hsieh, Deborah A. Wallach, Mike Burrows, Tushar Chandra, Andrew Fikes, and Robert E. Gruber. 2008. Bigtable: A Distributed Storage System for Structured Data. ACM Trans. Comput. Syst. 26, 2, Article 4 (June 2008), 26 pages. https://doi.org/10.1145/1365815.1365816

[3] Surajit Chaudhuri and Vivek Narasayya. 1997. An Efficient, CostDriven Index Selection Tool for Microsoft SQL Server. In Proceedings of the VLDB Endowment. VLDB Endowment.

[4] Databricks Engineering Blog. [n.d.]. Processing Petabytes of Data in Seconds with Databricks Delta. https://databricks.com/blog/2018/ 07/31/processing-petabytes-of-data-in-seconds-with-databricksdelta.html.

[5] Jialin Ding, Umar Farooq Minhas, Hantian Zhang, Yinan Li, Chi Wang, Badrish Chandramouli, Johannes Gehrke, Donald Kossmann, and David Lomet. 2019. ALEX: An Updatable Adaptive Learned Index. arXiv: 1905.08898

[6] Andrew Lamb et al. 2012. The Vertica Analytic Database: C-Store 7 Years Later. In Proceedings of the VLDB Endowment. VLDB Endowment.

[7] Mike Stonebraker et al. 2005. C-Store: A Column-oriented DBMS. In Proceedings of the 31st VLDB Conference. VLDB Endowment.

[8] Exasol. [n.d.]. The World's Fastest In-Memory Analytic Database. https://www.exasol.com/en/community/resources/resource/worldsfastest-analytic-database/.

[9] Volker Gaede and Oliver Günther. 1998. Multidimensional access methods. ACM Computing Surveys (CSUR) 30 (1998), 170-231. Issue 2.

[10] Alex Galakatos, Michael Markovitch, Carsten Binnig, Rodrigo Fonseca, and Tim Kraska. 2018. A-Tree: A Bounded Approximate Index Structure. CoRR abs/1801.10207 (2018). arXiv:1801.10207 http://arxiv.org/abs/1801.10207

[11] Jim Gray, Surajit Chaudhuri, Adam Bosworth, Andrew Layman, Don Reichart, Murali Venkatrao, Frank Pellow, and Hamid Pirahesh. 1997. Data Cube: A Relational Aggregation Operator Generalizing Group-By, Cross-Tab, and Sub-Totals. Data Min. Knowl. Discov. 1, 1 (Jan. 1997), 29-53. https://doi.org/10.1023/A:1009726021843

[12] Marios Hadjieleftheriou. 2014. libspatialindex. https: //libspatialindex.org/.

[13] Klaus Hinrichs. 1985. Implementation of the Grid File: Design Concepts and Experience. BIT 25, 4 (Dec. 1985), 569-592. https://doi.org/10.1007/BF01936137

[14] Andreas Hutflesz, Hans-Werner Six, and Peter Widmayer. 1988. Twin Grid Files: Space Optimizing Access Schemes. In Proceedings of the 1988 ACM SIGMOD International Conference on Management of Data (SIGMOD '88). ACM, New York, NY, USA, 183-190. https://doi.org/10.1145/50202.50222

[15] IBM. [n.d.]. The Spatial Index. https://www.ibm.com/support/ knowledgecenter/SSGU8G_12.1.0/com.ibm.spatial.doc/ids_spat_024. htm.

[16] Stratos Idreos, Martin L. Kersten, and Stefan Manegold. 2007. Database Cracking. Conference on Innovative Data Systems Research (CIDR).

[17] Stratos Idreos, Martin L. Kersten, and Stefan Manegold. 2009. Selforganizing Tuple Reconstruction in Column-stores. In SIGMOD. ACM.

[18] Stratos Idreos, Konstantinos Zoumpatianos, Brian Hentschel, Michael S. Kester, and Demi Guo. 2018. The Data Calculator: Data Structure Design and Cost Synthesis From First Principles, and Learned Cost Models. In ACM SIGMOD International Conference on Management of Data.
[19] Intel Corporation. 2014. Scaling Data Capacity for SAP HANA with Fujitsu PRIMERGY/PRIMEQUEST Servers. Technical Report.

[20] Irfan Khan. 2012. Falling RAM prices drive in-memory database surge. https://www.itworld.com/article/2718428/falling-ram-prices-drivein-memory-database-surge.html.

[21] Hideaki Kimura, George Huo, Alexander Rasin, Samuel Madden, and Stanley B. Zdonik. 2009. Correlation Maps: A Compressed Access Method for Exploiting Soft Functional Dependencies. Proc. VLDB Endow. 2, 1 (Aug. 2009), 1222-1233. https://doi.org/10.14778/1687627.1687765

[22] Tim Kraska, Mohammad Alizadeh, Alex Beutel, Ed H. Chi, Ani Kristo, Guillaume Leclerc, Samuel Madden, Hongzi Mao, and Vikram Nathan. 2019. SageDB: A Learned Database System. In CIDR 2019, 9th Biennial Conference on Innovative Data Systems Research, Asilomar, CA, USA, January 13-16, 2019, Online Proceedings.

[23] Tim Kraska, Alex Beutel, Ed H. Chi, Jeffrey Dean, and Neoklis Polyzotis. 2018. The Case for Learned Index Structures. In Proceedings of the 2018 International Conference on Management of Data (SIGMOD). ACM.

[24] Iosif Lazaridis and Sharad Mehrotra. 2001. Progressive Approximate Aggregate Queries with a Multi-resolution Tree Structure. In Proceedings of the 2001 ACM SIGMOD International Conference on Management of Data (SIGMOD '01). ACM, New York, NY, USA, 401-412. https://doi.org/10.1145/375663.375718

[25] Lin Ma, Dana Van Aken, Amed Hefny, Gustavo Mezerhane, Andrew Pavlo, and Geoffrey J. Gordon. 2018. Query-based Workload Forecasting for Self-Driving Database Management Systems. In SIGMOD. ACM.

[26] Donald Meagher. 1980. Octree Encoding: A New Technique for the Representation, Manipulation and Display of Arbitrary 3-D Objects by Computer. Technical Report.

[27] Microsoft SQL Server. 2016. Spatial Indexes Overview. https: //docs.microsoft.com/en-us/sql/relational-databases/spatial/spatialindexes-overview?view=sql-server -2017 .

[28] MonetDB. 2018. monetdb. https://www.monetdb.org/.

[29] G. M. Morton. 1966. A computer Oriented Geodetic Data Base; and a New Technique in File Sequencing (PDF). Technical Report. IBM.

[30] Vikram Nathan, Jialin Ding, Mohammad Alizadeh, and Tim Kraska. 2020. Learned Multi-dimensional Indexes. arXiv:1912.01668 https://arxiv.org/abs/1912.01668

[31] J. Nievergelt, Hans Hinterberger, and Kenneth C. Sevcik. 1984. The Grid File: An Adaptable, Symmetric Multikey File Structure. ACM Trans. Database Syst. 9, 1 (March 1984), 38-71. https://doi.org/10.1145/348.318586

[32] Beng Chin Ooi, Ron Sacks-davis, and Jiawei Han. 2019. Indexing in Spatial Databases.

[33] OpenStreetMap contributors. 2019. US Northeast dump obtained from https://download.geofabrik.de/. https://www.openstreetmap.org.

[34] Oracle Database Data Warehousing Guide. 2017. Attribute Clustering. https://docs.oracle.com/database/121/DWHSG/attcluster.htm.

[35] Oracle, Inc. [n.d.]. Oracle Database In-Memory. https: //www.oracle.com/database/technologies/in-memory.html.

[36] Yongjoo Park, Shucheng Zhong, and Barzan Mozafari. 2019. QuickSel: Quick Selectivity Learning with Mixture Models. In SIGMOD. ACM.

[37] Frank Ramsak1, Volker Markl, Robert Fenk, Martin Zirkel, Klaus Elhardt, and Rudolf Bayer. 2000. Integrating the UB-Tree into a Database System Kernel . In Proceedings of the 26th International Conference on Very Large Databases. VLDB Endowment.

[38] Felix Martin Schuhknecht, Alekh Jindal, and Jens Dittrich. 2013. The Uncracked Pieces in Database Cracking. Proc. VLDB Endow. 7, 2 (Oct. 2013), 97-108. https://doi.org/10.14778/2732228.2732229

[39] Scipy.org. [n.d.]. scipy.optimize.basinhopping. https: //docs.scipy.org/doc/scipy-0.18.1/reference/generated/scipy.optimize. basinhopping.html. 
[40] Dennis G. Severance and Guy M. Lohman. 1976. Differential Files: Their Application to the Maintenance of Large Databases. ACM Trans. Database Syst. 1, 3 (Sept. 1976), 256-267. https://doi.org/10.1145/320473.320484

[41] Hari Singh and Seema Bawa. 2017. A Survey of Traditional and MapReduceBased Spatial Query Processing Approaches. SIGMOD Rec. 46, 2 (Sept. 2017), 18-29. https://doi.org/10.1145/3137586.3137590

[42] Markku Tamminen. 1982. The extendible cell method for closest point problems. BIT 22 (01 1982), 27-41. https://doi.org/10.1007/BF01934393

[43] TPC. 2019. TPC-H. http://www.tpc.org/tpch/.

[44] Kostas Tzoumas, Amol Deshpande, and Christian S. Jensen. 2011. Lightweight Graphical Models for Selectivity Estimation Without Independence Assumptions. In Proceedings of the VLDB Endowment.
VLDB Endowment.

[45] Gary Valentin, Michael Zuliani, Daniel C. Zilio, Guy Lohman, and Alan Skelley. 2000. DB2 Advisor: An Optimizer Smart Enough to Recommend its own Indexes. In Proceedings of the 16th International Conference on Data Engineering. IEEE.

[46] Yingjun Wu, Jia Yu, Yuanyuan Tian, Richard Sidle, and Ronald Barber. 2019. Designing Succinct Secondary Indexing Mechanism by Exploiting Column Correlations. In Proceedings of the 2019 International Conference on Management of Data, SIGMOD Conference 2019, Amsterdam, The Netherlands, fune 30 - fuly 5, 2019. 1223-1240.

[47] Zack Slayton. 2017. Z-Order Indexing for Multifaceted Queries in Amazon DynamoDB. https://aws.amazon.com/blogs/database/z-orderindexing-for-multifaceted-queries-in-amazon-dynamodb-part-1/. 